\title{
Bone marrow transplantation for CD40 ligand deficiency: a single centre experience
}

\author{
K Khawaja, A R Gennery, T J Flood, M Abinun, A J Cant
}

\begin{abstract}
Background-CD40 ligand (CD40L) deficiency is a rare $X$ linked immunodeficiency disorder leading to recurrent bacterial infection, with cryptosporidial enteritis and subsequent hepatic cirrhosis. Bone marrow transplantation offers the only cure.

Objective-To analyse retrospectively the outcome of bone marrow transplantation for this condition in one centre.

Design-A retrospective case note analysis was performed, identifying all patients with CD40L deficiency who had undergone bone marrow transplantation between May 1988 and December 2000. Details of pre-existing infection, pretransplantation immunological and infective data, transplant procedure (particularly donor type and HLA match), conditioning regimen, and marrow manipulation were analysed. Post-transplantation data including infective episodes, engraftment details, immune function, complications, and outcome were recorded.

Results-Eight boys (age 1-14 years, median 5.75) had transplants. Six received $T$ cell depleted unrelated donor marrow. Four survive and have normal immune function. Six had previous Pneumocystis carinii pneumonia and three had histological liver damage. Survival was associated with younger age at transplantation and normal liver histology.

Conclusions-Bone marrow transplantation can be curative in CD40L deficiency. Better outcome is associated with younger age at transplantation and normal liver histology.

(Arch Dis Child 2001;84:508-511)
\end{abstract}

Keywords: CD40 ligand deficiency; bone marrow transplantation; immunodeficiency

Department of

Paediatric

Immunology,

Newcastle General

Hospital, Westgate

Road, Newcastle upon

Tyne NE4 6BE, UK

K Khawaja

A R Gennery

T J Flood

M Abinun

A J Cant

Correspondence to:

Dr Cant

email: a.j.cant@ncl.ac.uk

Accepted 21 March 2001 first year of life, recurrent bacterial sinopulmonary infections, and persistent Cryptosporidium parvum infections leading to sclerosing cholangitis, cirrhosis, and liver cancer. Other complications include autoimmune disease, neutropenia, and enteroviral meningoencephalitis. ${ }^{45} \mathrm{~A}$ European survey of CD40L deficient patients showed that $75 \%$ develop liver disease and only $20 \%$ survive into the third decade of life. ${ }^{6}$

Prophylactic co-trimoxazole and intravenous immunoglobulin replacement are the mainstays of treatment, together with advice to boil water to prevent cryptosporidium infection. Granulocyte colony stimulating factor (GCSF) can improve neutropenia. Nevertheless many patients given such optimal treatment still die of liver disease by early adult life. As this is a T cell disorder, at present only haemopoietic stem cell transplantation can cure the genetic defect.

Fasth reported the first allogenic bone marrow transplant in this condition, using an HLA matched brother as donor. ${ }^{7}$ There have been seven further case reports of successful bone marrow transplantation for $\mathrm{X}$ linked hyperIgM syndrome. ${ }^{8-15}$ However, bone marrow transplantation for immunodeficiency is not without risk, and for other primary immunodeficiencies such as the Wiskott-Aldrich syndrome, older age, ongoing serious infections, lung or liver disease, and lack of availability of a well matched donor are associated with a poor outcome after bone marrow transplantation.

We present the experience of bone marrow transplantation for CD40L deficiency in a single centre, with particular reference to the influence of pretransplant risk factors and donor type.

\section{Methods}

We studied retrospectively eight patients who received bone marrow transplants between September 1996 and December 2000, with follow up of between 0.5 and 3.5 years. The diagnosis was confirmed by demonstrating absence of CD40L on mononuclear cells separated on Ficoll, stimulated for 4 hours with phorbol myristate acetate $50 \mathrm{ng} / \mathrm{ml}$ and ionomycin $1000 \mathrm{ng} / \mathrm{ml}$, and stained with CD3 and CD40L antibodies. ${ }^{16}$ Mutations in the CD40L gene were also demonstrated by single strand conformation polymorphism analysis of individual exons of the CD 40 ligand gene. ${ }^{17}$

Before transplantation, patients were assessed by chest $x$ ray, computed axial tomography (CT) of the chest, pulmonary function testing, and bronchoalveolar lavage. Other investigations included measurement of serum 
albumin, alkaline phosphatase, bilirubin, $\gamma$-glutamyl transferase, and aspartate aminotransferase, a liver ultrasound scan, endoscopic retrograde cholangiopancreatography, and a liver biopsy with immunohistochemical staining.

Information was obtained about pre-existing infection and pretransplantation immunological and infective data, and details of the transplant procedure were recorded, particularly donor type and HLA match, conditioning regime, and marrow manipulation. The posttransplantation data recorded included description of infective episodes, engraftment details and immune function, complications, and outcome.

\section{PATIENT CHARACTERISTICS}

All eight boys had defective CD40L expression on activated $\mathrm{T}$ lymphocytes and a mutation in the CD40L gene. Six children had Pneumocystis carinii pneumonia (table 1); three required mechanical ventilation with additional extracorporeal membrane oxygenation in case 2 . Three had bronchiectasis on CT scan before bone marrow transplantation.

Pretransplantation bronchoalveolar lavage was negative in seven patients. A solitary Pneumocystis carinii cyst was found in case 7 , treated with intravenous cotrimoxazole before transplantation. Four patients had documented previous cryptosporidial infection and three had abnormalities on liver biopsy. None had hepatocellular carcinoma or oesophageal varices. There were no cases of cytomegalovirus infection pretransplantation. Five patients required G-CSF before transplantation. None had inflammatory bowel disease. Four had failure to thrive, one requiring total parental nutrition before transplantation for severe liver disease and chronic pancreatitis. All were receiving intravenous immunoglobulin replacement and had normal trough IgG levels.

Age at bone marrow transplantation ranged from 1 to 14 years (median 5.75 years). Standard European bone marrow transplantation working party cytoreductive conditioning protocols for $\mathrm{T}$ cell immunodeficiencies were followed. As conditioning for bone marrow transplantation, six patients received busulphan $16 \mathrm{mg} / \mathrm{kg}$ and cyclophosphamide 200 $\mathrm{mg} / \mathrm{kg}$. Patient 2 also receiving anti-LFA-1 antibody from day -3 to day +10 , and anti-CD2 antibody from day -2 to day+1, while patient 7 received fludarabine $30 \mathrm{mg} / \mathrm{m}^{2}$ from day -7 to day -3 , melphalan $140 \mathrm{mg} / \mathrm{m}^{2}$ on day -2 , and antithymocyte globulin 2.5 $\mathrm{mg} / \mathrm{kg}$ from day -6 to day -2 .

Two patients received sibling HLA identical whole marrows, while six received $\mathrm{T}$ cell depleted marrow from unrelated donors (table 2). Donor/recipient HLA A, B, C, DR, and DQ typing was performed by molecular DNA methods. Two of the $\mathrm{T}$ cell depletion studies were performed with CAMPATH ( $1 \mathrm{G}$ and $1 \mathrm{M}$, respectively) in vitro. The remaining four were performed by CD34+ positive stem cell selection (Miltenyi CliniMACS). All patients received cyclosporin as graft versus host disease prophylaxis. All were isolated under laminar flow and received thrice weekly oral co-trimoxazole (5 mg/kg twice daily) and weekly intravenous immunoglobulins. Patients known to have had cryptosporium infection received additional prophylaxis for this organism in the form of azithromycin and paromomycin; cases 3, 7, and 8 also received bovine serum colostrum.

\section{Results}

All patients had evidence of engraftment after transplantation. Full or partial chimaerism was demonstrated in all seven patients who were tested (table 2). All patients had evidence of infection during the transplant period (table 2). Three had ongoing cryptosporidial infections. Adenovirus type 5 was found in stool from patient 2 from day -1 , treated with oral and intravenous immunoglobulin and intravenous ribavarin. Human herpesvirus 6 was also detected in peripheral blood by polymerase chain reaction on day +55 , and treated with foscarnet.

Four patients are alive and well, with normal CD40L expression and no graft versus host disease. Two remain on intravenous immunoglobulin because they received their transplant less than a year ago. IgM levels are normal. Two patients off intravenous immunoglobulin have made adequate seroresponses to vaccination against tetanus, Haemophilus influenzae type $\mathrm{B}$, and pneumococcus.

Table 1 Patient characteristics

\begin{tabular}{|c|c|c|c|c|c|c|c|c|}
\hline \multirow[b]{2}{*}{ Clinical features } & \multicolumn{8}{|c|}{ Case number } \\
\hline & 1 & 2 & 3 & 4 & 5 & 6 & 7 & 8 \\
\hline Age at diagnosis (months) & 9 & 22 & 5 & 4 & 6 & 6 & 5 & 4 \\
\hline Age at transplant (years) & 1 & 3 & 4.5 & 7 & 4 & 13 & 14 & 14 \\
\hline Previous PCP & Yes & No & Yes & Yes & Yes & No & Yes & Yes \\
\hline Ventilated & No & No & Yes & No & Yes & No & Yes & No \\
\hline Thoracic HRCT & ND & Normal & Normal & Normal & Bronchiectasis & Normal & Bronchiectasis & Bronchiectasis \\
\hline Liver histology & ND & Normal & Normal & Normal & Normal & sc/cirr & Portal inflammation & sc/cirr \\
\hline ERCP & ND & Normal & Normal & Normal & Normal & $\begin{array}{l}\text { Dilated bile } \\
\text { ducts }\end{array}$ & Normal & sc \\
\hline Cryptosporidial enteritis & No & No & Yes & No & No & Yes & Yes & Yes \\
\hline Neutropenia & Yes & Yes & No & No & Yes & Yes & Yes & No \\
\hline G-CSF & Yes & Yes & No & No & Yes & Yes & Yes & No \\
\hline FTT & Yes & No & No & Yes & No & Yes & No & Yes \\
\hline
\end{tabular}

BMT, bone marrow transplantation; cirr, cirrhosis; ERCP, endoscopic retrograde cholangiogram; FTT, failure to thrive; G-CSF, granulocyte colony stimulating factor; HRCT, high resolution computerised tomogram; IVIG, intravenous immunoglobulin; ND, not done; PCP, Pneumocystis carinii pneumonia; sc, sclerosing cholangitis; TPN, total parental nutrition. 


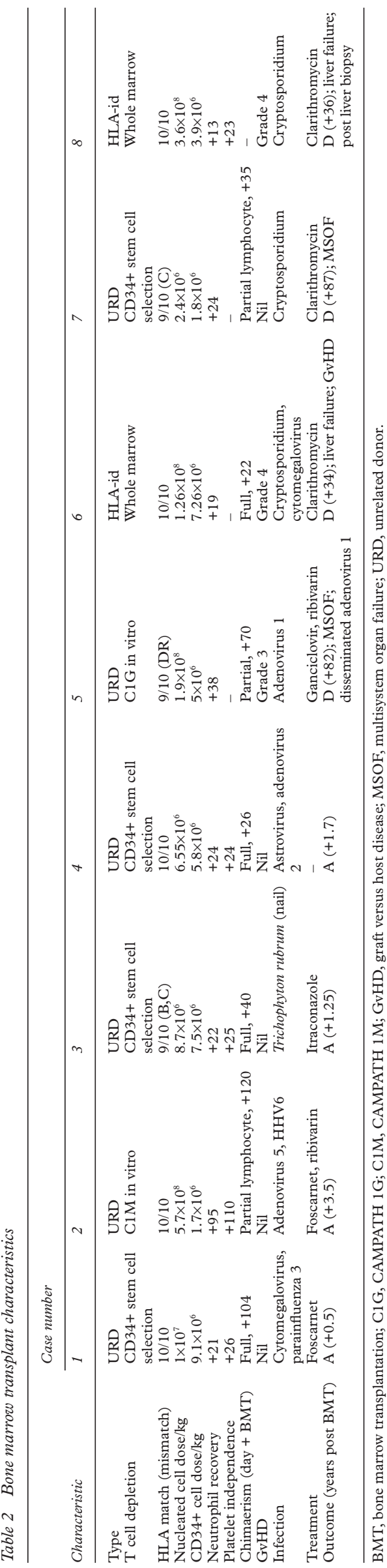

Four patients died. The unrelated donor for patient 5 was declared unfit on the day of harvest. A 9/10 substitution unrelated donor was found and harvested within 48 hours. The patient developed biopsy confirmed grade III acute skin and gut graft versus host disease on day 7, treated with high dose methylprednisolone for three days followed by antithymocyte globulin (rabbit) $5 \mathrm{mg} / \mathrm{kg} /$ day from day 16 to day 23. Adenovirus was detected in stool by ELISA from day 10 and treated with oral and high dose intravenous immunoglobulin infusion (12 g/week), ribivarin, and ganciclovir. By day 68 lung, liver, and gut disease was evident with adenovirus isolated from bronchoalveolar lavage, liver, and lower gastrointestinal tract. The child died of multisystem organ failure and adenovirus hepatitis on day 82 .

Patient 6 had severe liver disease before his bone marrow transplant, with diarrhoea, chronic cryptosporidial infection, sclerosing cholangitis, cirrhosis, and chronic pancreatitis; long term total parental nutrition was required before the transplant, with G-CSF for chronic neutropenia. He was treated with paromomycin, bovine serum colostrum, and ursodeoxycholic acid in addition to azithromycin. He died on day 34 from fulminant liver failure. Veno-occlusive disease, cryptosporidium infestation of bile ducts, biliary fibrosis, and graft versus host disease were demonstrated at necropsy examination.

Patient 7 had cryptosporidial ascending cholangitis with portal inflammation on liver biopsy. He developed severe cryptosporidial enteritis with massive diarrhoea despite treatment with azithromycin, paromomycin, bovine serum colostrum, and nitazoxamide. Capillary leak syndrome and pneumonitis led to multiorgan failure and death on day +87 .

Patient 8 had extensive bilateral bronchiectasis and severe liver disease before transplantation. Veno-occlusive disease requiring recombinant tissue plasminogen activator on day +9 and heparin infusion on day +11 complicated the post-transplant course, followed by severe progressive liver graft versus host disease. He died from haemorrhage following a liver biopsy while being assessed for orthoptic liver transplantation.

\section{Discussion}

The long term outlook for CD40L deficiency remains poor despite full supportive treatment with intravenous immunoglobulin replacement therapy, co-trimoxazole prophylaxis for Pneumocystis carinii infection, and G-CSF for neutropenia, together with prompt antibacterial treatment and nutritional support. Our results confirm that bone marrow transplantation can be curative and it had a successful outcome with $\mathrm{T}$ cell depleted mismatched unrelated donor bone marrow transplantation in four cases. However, bone marrow transplantation is risky and four patients died. Perhaps not surprisingly these were older patients with more advanced liver disease. Furthermore their deaths were all due to liver disease and occurred either because cryptosporidial infection progressed rapidly 
following pretransplantation cytotoxic conditioning therapy or because ongoing hepatic inflammation activated donor $\mathrm{T}$ lymphocytes, setting off severe liver graft versus host disease. Previous Pneumocystis carinii pneumonia did not appear to be associated with increased risk of death. Pre-existing liver disease appeared to be a greater risk factor than suboptimal donor matching, as the four survivors all received marrow from unrelated donors, whereas two of the four who died received marrow from HLA identical siblings. The number of patients in this series is small and caution is needed before drawing conclusions. Nevertheless these results suggest that, as in other primary immunodeficiency diseases like the Wiskott-Aldrich syndrome, bone marrow transplantation is more likely to be successful if performed in early childhood before significant liver damage has occurred.

For patients who already have significant liver disease, planning the best treatment programme remains a challenge, particularly as cryptosporidial infection cannot be eradicated despite the use of agents such as paromomycin, azithromycin, and bovine serum colostrum. Whether longer periods of anticryptosporidial treatment before bone marrow transplantation, with more drugs delivered intravenously or through a nasojejunal tube, would slow the rate of progression of cryptosporidial infection is unclear. Less damaging pretransplantation cytotoxic conditioning therapy might lessen the progression of cryptosporidial infection and liver inflammation, so reducing deaths from cryptosporidium and graft versus host disease. Regimens using fludarabine and melphalan hold promise ${ }^{18} 19$ and a recent report describes success with this method in children with CD40L deficiency and sclerosing cholangitis, ${ }^{20}$ but it should be noted that in our series, patient 8 was given such conditioning and still developed severe liver disease. Finally, the role of combined liver and bone marrow transplantation needs to be explored further, following the recent report of a successful case. ${ }^{13}$ As liver disease appears to recur rapidly in patients given a liver transplant without a bone marrow transplant it is likely that the two procedures should be undertaken within a short space of time.

Multicentre studies are needed for accurate assessment of pretransplantation risk factors, in particular detecting and quantifying cryptosporidial infection with new methods such as poymerase chain reaction, and assessing any consequent liver damage. ${ }^{21}$ It may be that less toxic conditioning regimens will make bone marrow transplantation less risky for older patients with significant damage. On the basis of our results so far we would advocate bone marrow transplantation at an early age before significant damage has occurred, when both HLA identical sibling and unrelated donor bone marrow transplantation can be successful. We are very grateful to Dr Alison Jones for performing CD40L
mutation analyses, Dr Gavin Spickett and Dawn Barge for permutation analyses, Dr Gavin Spickett and Dawn Barge for per-
forming flow cytometric analysis of CD40L expression, and $\mathrm{Dr}$ forming flow cytometric analysis of CD40L

1 Korthauer U, Graf D, Mages HW, et al. Defective expression of T-cell CD40L causes X-linked immunodeficiency with hyper-IgM. Nature 1993;361:539-41.

2 DiSanto JP, Bonnefoy JY, Gauchat JF, et al. CD40L mutations in X-linked immunodeficiency with hyper-IgM. Nature 1993;361:541-3.

3 Van Kooten C, Banchereau J. Functions of CD40 on B cells, dendritic cells, and other cells. Curr Opin Immunol 1997;9: dendritic

4 Andrews JF, Katz F, Jones A, et al. CD40L deficiency presenting as unresponsive neutropenia. Arch Dis Child presenting as ant

5 Cunningham CK, Bonville CA, Ochs HD, et al. Enteroviral meningoencephalitis as a complication of X-linked hyper IgM syndrome. 7 Pediatr 1999;134:584-8.

6 Levy J, Espanol-Boren T, Thomas C, et al. Clinical spectrum of X-linked hyper-IgM syndrome. $\mathcal{F}$ Pediatr 1997; 131:47-54.

7 Fasth A. Bone marrow transplantation for hyper-IgM syndrome. Immunodeficiency 1993;4:323.

8 Thomas C, De Saint Basile G, Le Deist F, et al. Brief report: correction of X-linked hyper-IgM syndrome by allogeneic bone marrow transplantation. N Engl F Med 1995;333:4269 .

9 Scholl PR, O'Gorman MRG, Pachman LM, et al. Correction of neutropenia and hypogammaglobulinemia in X-linked hyper-IgM syndrome by allogeneic bone marrow transplantation. Bone Marrow Transplant 1998;22:1215-18.

10 Bordigoni P, Auburtin B, Carret A, et al. Bone marrow ransplantation as treatment for X-linked immunodeficiency with hyper-IgM. Bone Marrow Transplant 1998;22:1111-14

11 Kato T, Tsuge I, Inaba J, et al. Successful bone marrow transplantation in a child with X-linked hyper-IgM syndrome. Bone Marrow Transplant 1999;23:1081-3.

12 Kawai S, Sasahara Y, Minegishi M, et al. Immunological reconstitution by allogeneic bone marrow transplantation
in a child with the X-linked hyper-IgM syndrome. Eur $\mathcal{F}$ Pediatr 1999;158:394-7.

13 Duplantier JE, Nelson RP, Ochs HD, et al. Successful bone marrow transplantation (BMT) for X-linked Hyper IgM (XHIM) syndrome. F Allergy Clin Immunol 1997;1:416.

14 Hadzic N, Pagliuca A, Rela M, et al. Correction of the hyper-IgM syndrome after liver and bone marrow transplantation. N Engl F Med 2000;342:320-4.

15 Gennery AR, Clark JE, Flood TJ, et al. T cell depleted bone marrow transplantation from unrelated donor for X-linked marrow transplantation from unrelated donor for X-linked 2000;137:290

16 Seyama K, Nonoyama S, Gangsaas I, et al. Mutations of the CD40L gene and its effect on CD40L expression in patients with X-linked hyper IgM syndrome. Blood 1998;92:2421-34.

17 Katz F, Hinshelwood S, Rutland P, et al. Mutation analysis in CD40L deficiency leading to X-linked hypogammaglobulinaemia with hyper IgM syndrome. Hum Mutat 1996;8: 223-8.

18 Giralt S, Estey E, Albitar M, et al. Engraftment of allogeneic hematopoietic progenitor cells with purine analogcontaining chemotherapy: harnessing graft-versusleukaemia without myeloablative therapy. Blood 1997;89. $4531-6$.

19 Slavin S, Nagler A, Naparstek E, et al. Nonmyeloablative stem cell transplantation and cell therapy as an alternative to conventional bone marrow transplantation with lethal cytoreduction for the treatment of malignant and non-
malignant hematologic diseases. Blood 1998;91:756-63.

20 Amrolia P, Gaspar HB, Hassan A, et al. Nonmyeloablative stem cell transplantation for congenital immunodeficienstem cell transplantation for
cies. Blood 2000;96:1239-46.

21 Davies EG, Amar C, Hadzic N, et al. Detection of cryptosporidium by polymerase chain reaction in patients with primary immunodeficiency [abstract]. Arch Dis Child 2000;82(suppl):A48. 